Itinéraires Itinéraires

Littérature, textes, cultures

2010-3 | 2010

Médiévalisme

\title{
L'aura du Moyen Âge sur la scène contemporaine
}

\section{Michèle Gally}

\section{OpenEdition}

\section{Journals}

Édition électronique

URL : http://journals.openedition.org/itineraires/1859

DOI : 10.4000/itineraires.1859

ISSN : 2427-920X

Éditeur

Pléiade

Édition imprimée

Date de publication : 1 novembre 2010

Pagination : 125-137

ISBN : 978-2-296-13150-7

ISSN : $2100-1340$

Référence électronique

Michèle Gally, "L'aura du Moyen Âge sur la scène contemporaine », Itinéraires [En ligne], 2010-3 | 2010, mis en ligne le 01 novembre 2010, consulté le 02 mai 2019. URL : http://journals.openedition.org/ itineraires/1859; DOI : 10.4000/itineraires.1859

\section{(c) (1) $\Theta$}

Itinéraires est mis à disposition selon les termes de la licence Creative Commons Attribution - Pas d'Utilisation Commerciale - Pas de Modification 4.0 International. 


\title{
L'aura du Moyen Âge sur la scène contemporaine
}

\begin{abstract}
Twentieth century dramatic transpositions of medieval matter, particularly Arthurian one, give various works which imply numerous questions about the aesthetic or ideological range of this choice of the Middle Ages. Indeed, there is a real difficulty, if not a wager, to translate medieval novels on a stage. We shall consider a theatrical experiment of a present topic: the settings of Merlin or the devastated country (written in 1981 by the German playwright Tankred Dorst) by Lavelli in 2005 and Dana in 2009. The dramaturgical choices of each producer, the differences between the creations and the text of the play, allow us to ponder on relations carried by contemporary artists with the idea of the Middle Ages.
\end{abstract}

Keywords : drama, Dorst, generic transposition, Middle Ages, modernism Mots clés : théâtre, Dorst, transfert générique, médiévalité, modernisme

On assiste au $\mathrm{xx}^{\mathrm{e}}$ siècle à une production relativement importante d'un théâtre à sujets médiévaux qui représente, pour le dire vite, en quelque sorte l'autre face de l'inspiration médiévale des romans de fantasy ${ }^{1}$. Les pièces qui nous arrêteront rompent, en effet, avec un théâtre religieux, ou plus largement, nourri de spiritualité chrétienne, tel que l'écrivit par exemple Claudel. Le coup d'envoi de ce type de théâtre fut peut-être donné par Gracq en 1948 avec Le Roi Pêcheur, au demeurant mal reçu par les spectateurs et la critique. Très peu joué, n'ayant jamais véritablement résolu ses impasses dramaturgiques, Le Roi Pêcheur est pourtant souvent convoqué, parfois en unique référence, comme une sorte de paradigme de la résurrection du Moyen Âge sur la scène théâtrale moderne. Or cette

1. Voir en particulier sur cette question Anne Besson, « Le mythe culturel en fiction : deux relectures de la préhistoire arthurienne par la fantasy contemporaine », dans Isabelle DurandLe Guern (dir.), Images du Moyen Âge, Rennes, PUR, 2006, p. 175-184; Anne Besson, La Fantasy, Paris, Klincksieck, 2007. 
résurrection ne passe pas, en l'occurrence, par un sujet médiéval historique (Jeanne d'Arc par exemple), mais par la réappropriation de figures et de récits appartenant à la tradition romanesque médiévale.

Ces œuvres dramatiques constituent, cependant, une sorte de corpus assez divers, tandis que le théâtre médiéval proprement dit demeure presque inconnu et presque totalement absent de la scène contemporaine. La plus récente expérience de mise en scène d'une pièce médiévale en France a été faite par Jacques Rebotier qui a présenté au Vieux Colombier en 2003 Le Jeu de la Fenillée d'Adam de la Halle. Cette expérience a été saluée de façon mitigée par la critique journalistique et le grand public ${ }^{2}$. On dira donc, pour schématiser, que l'on se trouve devant une sorte de situation paradoxale : le médiéval au théâtre est un texte contemporain, et un texte réécrit à partir de ce qui n'avait pas été pensé en termes dramatiques.

J'aimerais, dans cette perspective, verser une nouvelle pièce au dossier de ces transferts de la matière médiévale romanesque, et non pas dramatique, à la scène contemporaine. " Matière » sera à entendre comme le terme dont les médiévaux nomment la nébuleuse de récits potentiels qui existent en deçà de la forme que tel ou tel trouvère choisira de donner à celui qu'il décidera d'isoler, et d'exploiter, en fonction de son projet poétique ou idéologique. Par excellence, c'est la «matière de Bretagne », selon l'expression du trouvère Jean Bodel au début du XIII ${ }^{\mathrm{e}}$ siècle qui retient l'attention des écrivains et des dramaturges contemporains. Cette « matière », déjà qualifiée de «plaisante » et « vaine » par le trouvère médiéval, paraît encore disponible à tous les jeux d'illusions et de miroitements ${ }^{3}$. Centrale dans les réécritures narratives contemporaines, elle nourrit aussi toute une veine dramatique exigeante et proprement créatrice. Nouveau trouvère au sens plein, l'écrivain contemporain puise librement dans la «matière » pour lui conférer la forme qui lui semble convenir à son projet poétique et idéologique.

Pourquoi et comment se constitue cette nouvelle forme? Car les difficultés sont grandes à la mesure du double transfert opéré, historique et générique, le « théâtre » ouvrant désormais, plus qu'à l'époque médiévale, à une attente et une réception sans relation avec la lecture d'un roman ${ }^{4}$. Par ailleurs le texte de théâtre médiéval pose des problèmes spécifiques de reconnaissance et de traduction dramaturgique ${ }^{5}$.

2. Michèle Gally, « Résurrection du Jeu de la Feuillée. Une pièce de théâtre postmoderne », Littérature, $\mathrm{n}^{\circ} 148$ : Le Moyen Âge contemporain, perspectives critiques, décembre 2007, p. 10-27.

3. Prologue de la Chanson des Saisnes, éd. Annette Brasseur, Genève, Droz, 1989, 2 vol., v. 9.

4. Il n'y a pas lieu ici de parler de l'époque médiévale sauf à préciser que la définition d'une «théâtralité » effective des récits et des poèmes médiévaux est à comprendre plus largement que pour les époques suivantes. Voir à ce sujet Paul Zumthor, La Lettre et la Voix, Paris, Seuil, 1987.

5. Voir dans ce volume la contribution de Véronique Dominguez. 
Comment alors représenter, au sens de l'incarnation charnelle de personnages/acteurs, les héros romanesques médiévaux, déjà stylisés dans leur mise en fiction originelle, dont la résurrection minutieusement historique (des chevaliers en armes de quelle époque entre le $\mathrm{IX}^{\mathrm{e}}$ et le $\mathrm{XV}^{\mathrm{e}}$ ?) s'avère impossible et inutile sauf à rejoindre les apories d'un certain cinéma qui ne peut donner à voir que les clichés qu'il construit et dont il se nourrit. En outre l'espace même de la scène, lieu d'évolution de tels personnages, si grand soit-il, ne s'avère-t-il pas toujours trop étroit? Jorge Lavelli, metteur en scène en 2005 du Merlin ou la terre dévastée de Tankred Dorst, crée la pièce au théâtre romain de Fourvière à Lyon, et dit « resserrer » l'espace scénique au théâtre de Bobigny. Le souci de l'espace semble premier :

La réalisation d'un premier travail sur un lieu incomparable [...] m'a permis de sentir et de vérifier dans le concret la charge dramatique de l'ouvrage. C'est sur les bases de cette expérience que nous resserrons aujourd'hui dans le cadre tout aussi libre de la grande salle de la $\mathrm{MC}{ }^{6}$.

Il reste malgré cela vingt et un comédiens et trois musiciens à faire évoluer pour un «mastodonte théâtral comportant près d'une centaine de rôles ».

Un choix se pose donc au dramaturge, et au metteur en scène, entre un déploiement, en quelque sorte, mimétique du foisonnement narratif du roman médiéval arthurien, et ce que j'appellerai la métonymie d'une séquence condensée et partant, hautement dramatique comme chez Gracq. Ce choix rejoint de façon biaisée celui des médiévaux : écrire à l'infini (non une histoire sans fin mais un déploiement interne d'épisodes entrelacés) ou porter l'attention sur un épisode et un héros qui deviennent emblématiques de l'ensemble, selon deux options que se répartissent le roman en prose et le roman en vers. On retrouve une difficulté, ou une tentation, identique chez Florence Delay et Jacques Roubaud et leur Graal-Théâtre en vingt branches, écrit entre 1977 et 2005 ! Impossible à mettre en scène ${ }^{7}$ ?

Entre gigantisme et épure, reconstitution historique et signification métaphorique, quels enjeux motivent les dramaturges contemporains pour se mesurer à des obstacles presque insurmontables dans le passage du récit à la scène? Comment la référence au texte médiéval, qu'effectue le texte dramatique contemporain, constitue-t-il à la fois un renvoi au pré-texte (ces auteurs font un vrai travail sur les textes médiévaux) et fonctionnet-il comme un prétexte à parler d'autre chose que lui par le détour d'une exploration d'un passé qui, chez le spectateur, évoque le plus souvent un ensemble confus de clichés et, chez le spécialiste, suscite la tentation amère de compter les erreurs historiques et littéraires?

6. Texte du programme, octobre 2005.

7. Un essai a été fait par Marcel Maréchal au théâtre du Gymnase à Marseille en 1979 où furent jouées trois « pièces » de Graal-théâtre. 
Je ne pourrai pas, dans le cadre d'une seule contribution, établir une comparaison entre les trois œuvres qui me semblent baliser exemplairement cette tentative : Julien Gracq en 1948, Florence Delay et Jacques Roubaud en 1977 et 2005, Tankred Dorst en $1981^{8}$. Pour des raisons d'actualité théâtrale (Dorst a été mis en scène au théâtre de la Colline à Paris par Rodolphe Dana du 20 novembre au 19 décembre 2009 et le sera à nouveau à Bordeaux à l'automne 2010 par Nadia Fabrizio) je m'arrêterai presque exclusivement sur Merlin ou la terre dévastée. Parler pleinement du théâtre, texte et représentations, offre de réelles difficultés car le texte seul ne suffit pas et on ne saurait décrire par le menu une mise en scène et un spectacle. Mais, autour de cette œuvre de Dorst, le rassemblement d'un texte, désormais traduit en français, et de deux mises en scène très récentes, permet, de façon exceptionnelle, de réfléchir sur le double transfert qu'opèrent le dramaturge et le metteur en scène entre les récits médiévaux et leur théâtralisation, entre des personnages et une action appartenant à un passé lointain et leur actualisation à notre époque et sous nos yeux. Nous avons donc trois moments de la création dramatique, celui de l'auteur du texte, en l'occurrence un texte long et complexe, très littéraire, et ceux des deux metteurs en scène successifs. Les deux mises en scène ne présentent pas le même rapport au texte de Dorst et donc, au-delà de lui, à ce que l'on appellera la « médiévalité » ou le " médiévalisme ».

Je m'appuierai, dans le début de mon commentaire, prioritairement sur le texte de Dorst et, en arrière-plan, la mise en scène de Lavelli. Je reviendrai à la fin sur quelques réflexions que suscite pour moi le spectacle de Dana ${ }^{9}$.

\section{Choisir la matière médiévale : un geste difficile, un geste risqué}

Si l'on revoit brièvement ce que disent nos quatre auteurs (Gracq, F. Delay et J. Roubaud, Dorst) d'un tel choix, il apparait que non seulement celui-ci n'a rien d'évident mais qu'il constitue un geste de rupture. Dans sa préface, Gracq dit avoir trouvé dans les mythes médiévaux - celui de Tristan et celui du Graal - des «mythes non tragiques », des « histoires ouvertes » qui parlent non de punitions gratuites mais de «tentations

8. Tankred Dorst, né en 1925, auteur et dramaturge très connu en Allemagne, semble rencontrer une reconnaissance tardive en France : il a reçu en 2009 le prix européen de la littérature à Strasbourg. Son œuvre importante est peu traduite en France. Une de ses pièces les plus célèbres (Toller) a été montée par Chéreau en 1970. Merlin, traduit par Hélène Mauler et René Zand à l'Arche en 2005, semble, sur les pas de Jorge Lavelli en 2005, retenir l'attention des metteurs en scène.

9. Je n'ai pas pu participer aux rencontres entre le public et les metteurs en scène organisées par les théâtres. Mes réflexions sont donc entièrement spéculatives et n'engagent que ma lecture des spectacles auxquels j'ai assisté. 
permanentes et récompensées ${ }^{10} »$. La matière médiévale ne serait pas tragique en ce qu'elle serait pré-chrétienne en un temps où le seul tragique est le christianisme. Gracq pose un paradoxe surtout si l'on considère le caractère désespéré du Roi Pêcheur et l'échec de son Perceval.

Aux antipodes, apparemment, F. Delay et J. Roubaud se déclarent d'abord séduits par le succès "populaire » de l'adaptation théâtrale du Roland Furieux de l'Arioste (mise en scène de Luca Ronconi en 1973) et cherchent un équivalent médiéval et français malgré le rapport différent entretenu par les Italiens et les Français aux textes médiévaux. Parlant du Graal, ils le qualifient à leur tour de «mythe des mythes, symbole du symbolisme... »; si « la religion chrétienne s'y accroche, il la dépasse ». Les romans du Graal constitueraient une fusion des mythes païens des origines, des métamorphoses, des objets rituels du Mystère chrétien, du roman familial de la chevalerie ${ }^{11}$.

Le médiéval est compris d'abord comme un syncrétisme de récits puisant à plusieurs mythologies. Cette lecture constitue une rupture par rapport à celle faite plus communément $\mathrm{au} \mathrm{XIX}^{\mathrm{e}}$ et au $\mathrm{XX}^{\mathrm{e}}$ par des auteurs pour qui l'époque médiévale était par excellence (avant les Lumières et la Révolution) le temps triomphant et unifié de la Foi. La vision d'un Occident chrétien, indéniablement historique, se fissure et, ce qui est désormais mis en exergue, c'est ce que les récits médiévaux charrient de non chrétien. Cette laïcisation s'allie à un déplacement de la tragédie (précisément chrétienne) vers l'utopie, plus exactement l'histoire des utopies, car la matière arthurienne, c'est-à-dire l'histoire du royaume arthurien, est alors saisie dans sa dimension humaine, non pas divine mais politique. Or les utopies politiques (du moins chez Dorst qui a vécu l'époque nazie) sont ressenties comme les grands drames du $\mathrm{xx}^{\mathrm{e}}$ siècle. $\mathrm{Au}$ désir de trouver une esthétique non classique (présent chez tous nos auteurs), une esthétique qui serait moderne, se mêle l'effort pour universaliser les figures médiévales, plus exactement, les deshistoriser. Les chevaliers deviendraient, en un renversement hardi de leur altérité, des miroirs possibles des hommes contemporains, contemporains compris moins dans leur dimension individuelle que collective. Qu'ils optent pour une version ironique et ludique comme F. Delay et J. Roubaud, ou violente et tendue comme Gracq, ou pour un mélange des deux comme Dorst, nos auteurs déclarent ne parler que de notre époque. Or, les héros arthuriens (même chez Gracq) ne constituent pas, alors, des modèles, comme ceux de la tragédie classique, mais des figures brouillées, négatives, voire grimaçantes, de nous-mêmes. La richesse foisonnante du matériau narratif arthurien semble apte à traduire la complexité autoproclamée et la perte de

10. Julien Gracq, Le Roi Pêcheur, Paris, José Corti, 1989, p. 10.

11. Florence Delay, « Graal soixante-Treize », dans Michèle Gally (dir.), La Trace médiévale et les écrivains d'aujourd'hui, Paris, PUF, 2000, p. 42-43. 
sens du monde moderne. Par ailleurs, la légende offre un parcours complet d'une origine (la création du royaume) à une disparition (son effondrement final et définitif). D'un blanc à un blanc. On y passe, en effet, d'une lutte féroce entre deux dragons mythologiques représentant les guerriers qui se battent pour le pouvoir, au duel mortel d'un père et d'un fils trop humains pour ce même pouvoir. Le rayon qui passe à travers la blessure du fils frappé par son père ou la main qui saisit l'épée Escalibur dans le lac (la mer chez Dorst), voire la barque qui emporte Arthur à Avalon, ne restent dans l'esprit que comme des artifices qui servent à atténuer le choc. F. Delay et J. Roubaud, fidèles à leur option oulipienne, s'efforcent d'effacer le drame en mettant en vedette dans la dernière branche celui qui a écrit l'ensemble, Blaise $^{12}$. Tout ne serait-il que littérature ${ }^{13}$ ? On en doute. Dorst pour sa part déclare avoir commencé par écrire la fin.

La difficulté, en effet, qui constitue aussi ce que l'on peut appeler, au sein de cette histoire de morts, la vitalité des récits arthuriens, tient, on l'a dit, au nombre et à la répétition d'épisodes semblables et différents, à la combinaison presque infinie des parcours des personnages. Cette difficulté à les embrasser devient difficulté à les récrire. Hormis Gracq, nos dramaturges ambitionnent d'y parvenir, ils ne choisissent pas. Les metteurs en scène, eux, en revanche choisissent. Ces choix, plus importants chez Rodolphe Dana qui abrège beaucoup la pièce, révèlent leur interprétation et les enjeux qu'ils poursuivent. Le texte de Dorst est, à bien des égards, en effet, impossible à mettre en scène, la matière arthurienne constituant par elle-même un défi à sa transposition.

Dorst commence, avoue-t-il, par s'ennuyer en lisant Malory ${ }^{14}$, il ne sait que faire de tous ces récits. Puis il découvre une halle aux poissons dans le port de Hambourg : le lieu produira l'œuvre nouvelle, il permettra

12. Blaise, très présent par ailleurs dans la mise en scène de Rodolphe Dana, n'est pas une invention de Dorst. Dans le roman médiéval de Merlin, Blaise est le confesseur de la mère de Merlin et devient le scribe de celui-ci qui lui dicte les événements (guerres, apparition d'Arthur, établissement de la Table Ronde, etc.) qu'il orchestre lui-même. Nous lisons donc d'une certaine manière (car le montage narratif médiéval est plus compliqué) le roman de Blaise.

13. Cette interrogation n'est pas absente de la pièce mais elle s'exprime davantage à travers Merlin. Excédé par la vision des horreurs qu'infligent les hommes à d'autres, vision due à sa nature diabolique d'omniscience, Merlin, par exemple, s'écrie : « Je suis un artiste, qu'est-ce que j'en ai affaire! » (p. 138). Dans la pièce de Cocteau, Les Chevaliers de la Table Ronde, Merlin enchante de façon négative la Bretagne arthurienne; il est une figure de faussaire mais aussi un double possible de l'auteur dramaturge. Voir à ce sujet l'article de Fabienne Pomel, «Figures du faussaire et de l'enchanteur dramaturge. Réécriture, falsification et théâtralité dans Les Chevaliers de la Table Ronde de Cocteau ", Cahiers de Recherches Médiévales, $\mathrm{n}^{\circ} 18,2009$, p. 367-386. Cette fonction se retrouve différemment chez Dorst.

14. Thomas Malory (Morte Darthur, 1485), chevalier anglais qui récrivit en les compilant et les transformant parfois les récits arthuriens. Il les a popularisés en Angleterre et a ouvert la voie à la littérature « arthurienne » surtout anglo-saxonne du Xvi $\mathrm{l}^{\mathrm{e}}$ au $\mathrm{Xx}^{\mathrm{e}}$ siècles. Dorst dit avoir lu aussi Wolfram d'Eschenbach (Parzival) et Chrétien de Troyes. 
sa mutation dramaturgique ${ }^{15}$. L'espace, concret sous l'œil du dramaturge, serait donc premier, s'y projette l'entrecroisement structural des épisodes que F. Delay et J. Roubaud traduisent dans une écriture en branches. C'est à partir de la découverte d'une " grandeur » que la pièce, soudain devenue possible à traduire sur une scène, peut s'écrire : «Merlin dans une halle, une longue pièce pour un large public - voilà qui vous contraint à une certaine écriture, à une certaine conduite des scènes, à l'utilisation de certains moyens. Il faut élargir, trouver le rythme. »

De cette véritable transposition spatiale du texte médiéval, surgit et se façonne un texte de théâtre, à partir de ses contraintes propres, en particulier celle de se rendre visible, de transformer des actes et des émotions narrés en spectacle pour la vue et pour l'ouïe. À travers ce gigantisme assumé, le travail de Dorst détache l'ensemble du désespoir sombre d'un roi Pêcheur, l'inverse presque. En témoignent les réactions au spectacle de Lavelli que l'on lit dans la presse. Ainsi Cazaux, dans Le matricule des anges, rendant compte du spectacle de 2005, multiplie les adjectifs " énorme », «formidable », « foisonnante» pour dire : " $\mathrm{Ce}$ qui est particulièrement réjouissant, c'est la vitalité avec laquelle Dorst procède avec ses personnages. Ca crie, ça gueule, c'est énorme et souvent très drôle ${ }^{16}$. »

Entre dérision et burlesque, entre Shakespeare et Beckett, le drame arthurien sera devenu jubilation théâtrale, quelque chose qui rappellerait à la fois Rabelais et Bruegel, nourrissant une esthétique théâtrale aussi éloignée de la scène classique que du roman médiéval. Dans ce mélange de vie et de mort, Dorst ne cherche-t-il cependant qu'à faire rire?

\section{Comique ou pathétique? Une dramaturgie subtile et fragile}

Le début de Merlin coïncide avec l'entrée en scène d'un clown qui s'avèrera être l'oncle de Merlin : « brute massive, entre en boitant, s'arrête, regarde autour de lui en ouvrant de grands yeux, il est suivi de sa sœur, enceinte, une géante ${ }^{17}$. $\gg$ Les personnages se disputent à cris stridents, coups et chutes se multiplient et surtout s'engage une série d'interpellations de (faux) spectateurs car le clown est à la recherche de l'homme qui a engendré l'enfant que porte sa sœur. Tout le monde est suspecté, un spectateur est giflé et plusieurs gags s'enchaînent : un monsieur bien disposé à adopter l'enfant s'enfuit devant le dévoilement grotesque de la nudité de Berthe la géante. Rothschild est accusé d'être le père et se débarrasse difficilement du clown. À ces trucs comiques et assez grossiers succède l'accouchement

15. Entretien de Peter Von Becker avec Tankred Dorst, 1979, extraits reproduits dans Merlins Zauber, Francfort-sur-le Main, Suhrkamp Verlag, 2001 (voir Théâtre-contemporain.com). Sauf indication contraire c'est de ces discussions que je tire quelques remarques de Dorst.

16. Le matricule des anges, $\mathrm{n}^{\circ} 65$, juillet-août 2005 .

17. Tout ce début est supprimé par Rodolphe Dana. 
de Berthe : une nuée de démons s'abat sur la scène, ombre et lumière se succèdent, tandis que le clown se roule en fœtus dans un coin. Ses cris et ses gémissements se mêlent à ceux de la parturiente. Enfin, soudain, Merlin apparaît en homme nu, barbu, qui apprend rapidement à se tenir droit et à marcher puis se met à lire le journal.

Ces effets n'installent pas une tragédie mais une parade et une ambiance de cirque. En son début, la pièce, selon une manière fréquente dans le théâtre du $\mathrm{Xx}^{\mathrm{e}}$, se joue au centre d'échanges entre les deux espaces, poreux, du public et de la scène, elle se place aussi au carrefour des temps : À Rotschild succèdera Mark Twain qui accompagnera un moment les chevaliers arthuriens en critiquant leurs paroles ${ }^{18}$. Les spectateurs en pères potentiels ne renvoient-ils cependant qu'à un jeu de scène facile, une captatio benevolentiae, ou inaugurent-ils une forme d'implication, d'interaction entre le monde présent et le monde non tant passé que, au sens propre, illusoire de la scène? Les lumières et les cris dominent ce début et des chutes ou des coups résonnent sans être vus. L'arrivée du vrai père, le diable, renforce le heurt entre les gestes clownesques et les fulgurances d'une soudaine violence : la bague du diable laisse une marque sanglante sur le visage du clown. C'est le premier « sang » qui ouvre à une peur à la fois ridicule et sérieuse. On entre peu à peu dans un drame indécis. À la fin de la scène des dragons et de l'usurpateur Vortigern (suivant exactement le roman de Merlin) le clown est soudain paralysé :

il opine du chef, ouvre la bouche pour répéter «tu gagnes » mais aucun mot n'en sort, il jette la tête en avant, vomit un flot de sang. Vortigern le frappe.

Le clown crie : «Aïe, je n’y peux rien! C'était Merlin! Merlin m'a ensorcelé! »

Merlin : « une abominable lumière

De sang baigne

L'effroi du monde ${ }^{19}$. »

18. Dans les premières scènes Mark Twain joue celui qui interroge, du point de vue d'une époque moderne rationaliste, les chevaliers sur leurs agissements et en particulier il traite Merlin de simple « illusionniste » : « ... je veux dire [...] qu'il n'existe rien qui ne s'explique scientifiquement, et que tout ce que je viens d'entendre semble indiquer qu'il s'agit d'une personne qui a l'art et la manière d'épater avec des combines apparemment inexplicables, et peut-être de mystifier ainsi les candides... » (p. 49). Il est très mal accepté par les chevaliers Sa présence s'estompe pour resurgir dans un bref moment où il se trouve en compagnie d'Arthur et où il demande aux spectateurs : « vivons-nous à l'Âge d'Or? » (p. 142). Ce personnage a une fonction de mise à distance de toutes les croyances, celles des médiévaux, celles des contemporains. Représentant en outre un scientisme dépassé, il souligne que rien ne constitue une référence pour parler du monde. Il n'est pas repris par Rodolphe Dana.

19. Il s'agit d'une citation de William Rowley, poète anglais du XVII siècle. Des extraits diversement poétiques émaillent la pièce (p. 43). 
Plus loin, Berthe reviendra au sein de la troupe grotesque et effrayante qui deviendra aussi celle de Mordred et accompagnera, en écho, les agissements cruels et sadiques de celui-ci et de ses frères. Ils tuent sauvagement le chien de Galaad, puis, à l'issue d'une véritable séance de torture, leur propre mère, Morgause, et son vieil amant, le chevalier Lamorak. Le sang de cette scène terrible se mêle au maquillage de clown dont Mordred a peint Lamorak dans un geste de dérision et d'humiliation.

Ces quelques exemples, qui seraient à poursuivre, montrent comment se met en place un entrelacement serré entre burlesque et violence, de plus en plus inquiétant. Aucune tension, cependant, ne s'installe entièrement sans que la scène suivante ne la rompe ou ne dévie le spectateur vers d'autres registres, d'autres personnages différents ou opposés aux précédents. Un discours familier et moderne alterne avec des récitations de poèmes et de chansons de toutes époques et de toutes langues. Le registre familier et prosaïque se retrouve de la même manière dans Graal-théâtre, ainsi que les citations d'œuvres diverses, mais il ne s'y accorde pas avec une violence soudain mise à nu. Il ne joue pas d'une autre discordance que celle d'un contemporain mêlé au médiéval: clin d'œil aux spectateurs plus que travail contrapuntique entre les registres comme chez Dorst. Chez celuici, le spectateur est bousculé, jamais paisible car il lui est impossible de s'installer dans une atmosphère. La pièce ne l'interpelle pas seulement avec les faux spectateurs du début, ou la dissonance d'une référence reconnue, mais par une déstabilisation constante. Par là il entre dans le récit médiéval qui lui est représenté. Il a quelque chose à voir avec lui. Dorst parle du théâtre en des termes proches de la catharsis aristotélicienne :

Je pense que le théâtre est l'une des plus grandes inventions de l'humanité, au même titre que la roue, ou que l'usage du feu. Tant qu'il $\mathrm{y}$ aura des hommes, ils se joueront quelque chose et, en se jouant quelque chose, ils diront: je suis ainsi, tu es ainsi... Sur la scène du théâtre, la question de la situation de l'homme est toujours de nouveau posée. Et c'est ainsi que je vois le théâtre comme institution faite non pour édifier mais pour sauver les êtres humains ${ }^{20}$.

À la faveur de l'orchestration d'un médiéval en partie attendu et reconnaissable par les spectateurs, Dorst produit du grand spectacle qui n'est pas seulement de divertissement. La mise en scène de R. Dana paraît, au contraire, s'éloigner de cette préoccupation et privilégier le seul spectacle. J'y reviendrai.

Mieux peut-être que Gracq ou F. Delay et J. Roubaud, le dramaturge allemand nous met en présence de la légende médiévale, non pour que nous la connaissions et en devenions savants, mais pour, à travers elle, toucher notre présent. Celui-ci rejoint le passé parce que l'un et l'autre, 
quelles qu'en soient les manifestations historiques, actualisent l'essentiel, seul pérenne, à savoir notre condition humaine. Là se joue le problème central de ce rapport (re)créatif au Moyen Âge. Nous sommes au cœur des motivations de ces emprunts à un passé largement ressenti comme légendaire. On retrouve, de façon détournée et impropre, les outils forgés par Georges Didi-Huberman via Wartburg pour l'histoire et l'analyse de l'art : feuilletage de temps hétérogènes qui se fondent en une œuvre, notion d'empreintes, d'anachronisme fécond, qui font remonter pour le spectateur un/des passé(s) propre(s) à retravailler son présent en un retour que l'on qualifiera d'impur, de mêlé, d'anhistorique ${ }^{21}$. Ce retour sera perçu ou non par les spectateurs, sera voulu ou non par le metteur en scène, il sera, ou non, le vecteur d'une catharsis théâtrale. La question rebondit cependant : pourquoi choisir le médiéval? Question qui, pour les metteurs en scène, se pose à un autre niveau encore : pourquoi choisir de représenter un texte dramatique aussi dense et insaisissable, aussi long et, pour tout dire, aussi touffu que celui de Dorst?

Les pièces à sujets médiévaux sont rarement des succès. Il y a une difficulté réelle à représenter les personnages médiévaux sur une scène, alors même que les textes de ces pièces peuvent être des réussites littéraires - je ne refais pas la liste des échecs depuis Gracq. Chez T. Dorst, des passages entiers, outre la longueur du texte et le nombre de ses personnages, semblent défier une dramaturgie et relever davantage de la narration. Mais il trouve, et crée, en Merlin un personnage susceptible de contrôler, au sens propre, la théâtralité de l'ensemble.

\section{Achronie/uchronie. Merlin le prestidigitateur}

Il n'y a pas, face à ceux de Tristan et du Graal, de mythe de Merlin. Plus exactement, le personnage appartient à celui du Graal ou à celui d'Arthur. Dorst a dit dans des interviews que le personnage de la légende arthurienne qui l'intéressait le plus, c'est-à-dire qui éveillait le sentiment d'une proximité en lui, était Perceval. Il écrira une pièce sur lui, en 1987, précédée de deux récits : un scénario, Le Sauvage et L'Homme nu. Ces textes réfléchissent sur la sauvagerie (Perceval est « naïf », « non éduqué ») et la société quand la première se mue, par idéalisme, en brutalité aveugle et irréconciliable avec la société, en marginalité. Envisagée ainsi, la sauvagerie permet un retour réflexif sur les valeurs du bien et du mal, sur les notions

21. Plusieurs travaux de Didi-Huberman peuvent être rappelés au croisement de sa réflexion sur les temps et la réception des œuvres, mais aussi de sa discussion de la notion d' « aura » chez Benjamin et de son dialogue à son propos avec Giorgio Agamben. On se contentera de signaler Devant le temps, Paris, Minuit, 2000. Ces outils, féconds, sont cependant à reconsidérer pour la lecture des textes, y compris, me semble-t-il, du théâtre, qui est un travail second à travers la lecture/interprétation du metteur en scène du texte d'un auteur. Le rapport du spectateur à ce qu'il voit ne saurait être celui du spectateur d'un tableau. 
de l'amour et de la mort, sur les possibilités de construire des liens sociaux. Perceval est présent dans Merlin, il participe, différemment de Mordred, d'une remontée de la violence qui menace le royaume. Sa «sauvagerie » terriblement mise en scène par le dépeçage atroce (ou ridicule?) du chevalier vermeil, sauvagerie comme naïve, sans moyen de se penser, cette sauvagerie se retourne contre les autres et contre lui-même, contre un monde dont il ne voit plus, lui l'enfant heureux de la nature, que l'envers " gaste ${ }^{22}$. C'est grâce à Merlin, se transformant en Trévizent, le roi du graal devenu un flagellant sanguinolent, que s'opère un nouveau retournement et que Perceval revoit les prairies, les fleurs, bientôt Blanchefleur.

Mais Dorst a, en 1981, choisi Merlin, un fils de diable désobéissant à son père qu'il craint, un prestidigitateur et un illusionniste dont il fait surtout un désillusionniste. "Merlin oder das würste Land» : que comprendre dans ce double titre, des termes complémentaires ou opposés? La « terre gaste » des romans arthuriens, damnation d'une obscure faute et d'un Graal non interrogé, n'est pas la Nature et Merlin n'est pas simplement la Société qui s'y opposerait. Il est le démiurge de la forme politique que peut prendre celle-ci, il est, dans la pièce, celui qui mène les personnages, celui qui fait les scènes : il est le roi Pêcheur, il est Trévizent, etc. Rien n'existe en dehors de ce qu'il fabrique sous nos yeux et ceux des personnages qui l'entourent. Mais, dit-il, il ne peut contraindre ni surveiller ceux-ci. Et il n'y a de destin que celui que chaque homme se fait. L'existence et non l'essence. Là se tient le propos moderne que permet d'exemplifier, en une sorte d'allégorisation, le personnage légendaire. Merlin n'a pas d'être stable, il n'est que métamorphose, adaptations successives à ses actes et aux situations qu'il traverse.

Il est choisi parce qu'il est, non seulement, le maitre d'œuvre mais aussi le metteur en scène d'une fable de l'Histoire, celle du royaume d'Arthur. Un metteur en scène présent dans la pièce ${ }^{23}$. Merlin est aussi, par sa nature diabolique, un être hors du temps, qui appartient à tous les temps et à aucun, un visionnaire du passé et du futur. Et il nous tend la main par-dessus les siècles, par dessus le «quatrième mur» de la scène théâtrale, construisant et déconstruisant sans cesse l'illusion dans laquelle sont piégés les personnages et avertissant les spectateurs de s'en tenir à distance. L'illusion théâtrale, exhibée, dénonce l'illusion utopiste. L'esprit des hommes construit des artefacts que leurs passions détruisent ou qui les tuent. Mais ils ne peuvent s'en empêcher et l'Histoire se poursuit comme le spectacle continue. Le théâtre paraît l'art le plus propre à mettre en abyme cette dynamique. Dorst donne une leçon d'histoire et de théâtre.

22. Perceval à Gauvain : «Qu'est-ce qui t'a amené dans cet affreux désert? / — De quel désert parles-tu? / - Dans cet affreux désert de pierres et d'éboulis, avec cet arbre en feu, tout seul là-bas? / - Je ne vois pas d'éboulis. Tu parles du marronnier avec ses fleurs comme des chandelles rouges? » (p.164).

23. Les effets de théâtre dans le théâtre sont très présents dans les pièces de Dorst. 
Merlin saute et danse, il se mêle aux demoiselles, représentées en girls de revue, du château de la Merveille conquis par Gauvain. Il se trouve partout. En ce sens il serait une possible figure du dramaturge, comme chez Cocteau, autant que du metteur en scène, un dramaturge qui prend les traits d'un simple prestidigitateur de cabaret ${ }^{24}$, à la fois ludique, inquiet et désabusé ${ }^{25}$. Mais, quand tout est consommé, Merlin, éternel, chante, sur la scène vidée, d'une « voix haute et magnifique ».

Au-delà cependant de la rencontre d'un dramaturge, politique et humaniste, et d'une figure légendaire non humaine, pourquoi le Moyen Âge? Question qui fait encore et toujours retour et à laquelle le dramaturge et les metteurs en scène proposent, à travers leur travail respectif, des réponses différentes.

Loin de toute reconstitution d'une époque, le théâtre à sujet médiéval auquel émarge cette œuvre, renvoie à un lieu temporel inassignable, une uchronie qui peut être rapprochée de celle des mythes sauf que ce brouillage des temps, associé directement à la figure légendaire de Merlin, fait que ces histoires sont portées jusqu'à nous mais sans identification clairement possible pour nous. Il ne s'agit pas «d'apprivoiser le mythe » (comme le disait Gracq) mais de le maintenir à distance dans son étrangeté. J'ai noté plus haut quelques procédés de distanciation mis en place par Dorst. Or nous, spectateurs, résistons aussi, à partir de notre appréhension d'un tel passé. Nous n'appartenons pas à ces temps sauvages même si, le temps d'une représentation, ils nous appellent à une reconnaissance grinçante, déformée, terrible et grotesque que nous refusons. Magie et violence se mêlent sur cette autre scène où, silhouettes fantasmatiques et cauchemardesques, les figures médiévales évoluent à la faveur d'une quincaillerie (paganisme, christ et diable mêlés) tonitruante.

Car la rencontre avec le Moyen Âge et ses héros déchus se fait à travers une esthétique (ou une écriture) qui est la nôtre, celle de la plus extrême modernité. C'est par ce détour que le médiéval innerve le présent de nos représentations. En ce point, les choix de Rodolphe Dana privilégient, de manière plus appuyée que chez Lavelli, une dérision généralisée, assument un bric-à-brac, donné pour tel en spectacle et produisant le spectacle. Sans jouer sur les mots, le "médiévalisme » rejoint là, plus que la modernité (qui est celle de l'écriture de Dorst), le « modernisme » actuel ${ }^{26}$.

24. Devant Viviane, qui veut apprendre la magie, il fait surgir un lapin (p. 192).

25. À la fin d'une discussion avec le diable où il réfute l'existence du Bien et du Mal, tandis que le diable se réjouit de tous les idéalistes qui mènent les gens droit chez lui en enfer, Merlin s'écrie : « Je ne veux plus! Je ne veux plus rien avoir à faire avec la maudite histoire du monde! » (p. 187).

26. J'entends par ce terme, le style esthétique propre à l'époque moderne dans ses évolutions les plus actuelles, en ce qu'il réfute idéal et beauté, en ce qu'il se construit à partir de matériaux hétéroclites, bas ou précieux, tous mêlés, dans un refus de choix et de hiérarchie. En cela le terme ou la notion de « médiévalisme », que le colloque nous invitait à considérer, 
La modernisation formelle, poétique, se fait, nous l'avons brièvement montré, par toutes sortes d'interpolations, de citations, d'anachronismes de plus ou moins bon goût, l'ensemble aboutissant à un texte hybride, composite, en un mot, monstrueux. Car la réécriture de ces textes anciens, généralement peu connus dans leur lettre par le public, se fait volontiers à travers des éclats brisés, des scènes, des tableaux, où il est aussi de brefs moments de grâce (joie enfantine de Perceval, amour de Lancelot et de Guenièvre, mélancolie d'Arthur...). Or c'est de cet inachèvement, de la laideur prononcée dont les contemporains habillent la matière médiévale, de cette dysharmonie, qui lui est étrangère, qu'elle ferait signe vers notre modernité, par-delà le rêve de beauté, fût-il tragique, du classicisme, ce sublime auquel notre époque, éclatée, pessimiste et désabusée, n'adhère plus. La fin des utopies, la fin de toute spiritualité, nous laissent seuls avec nos ridicules et nos bassesses. Autant en rire surtout par le truchement d'ancêtres aussi mal dégrossis. On est proche de la version des Monthy Python, ou plus récemment du feuilleton Kaamelott d'Alexandre Astier, proximité dont témoignent les quelques mots, familiers et parfois crus, avec lesquels chaque acteur parle de son personnage dans le programme du théâtre de la Colline. Chez Rodolphe Dana, alors que tout ou presque suscite le rire, tout manque du moindre horizon, de la moindre échappée sur un rêve. Les coupes qu'il fait dans le texte de Dorst concourent à cet effet. La minoration du rôle de Merlin ôte à l'ensemble encore un peu de son liant, de sa cohérence. Merlin ne chante plus à la fin; il a disparu. Dana va jusqu'au bout de la logique de l'esthétique contemporaine, que je qualifie de moderniste. C'est là la cohérence d'une mise en scène au demeurant dynamique, qui marche bien : on ne s'ennuie pas à son spectacle et ses acteurs ne ménagent pas leur peine. Leur laideur volontaire et celle des costumes, la vulgarité triomphante des personnages et des situations, accentuent le côté farcesque et évacuent toute tragédie quand la quête d'idéal s'avère simplement ridicule (à travers Perceval) et que triomphent les appétits des corps et des pulsions (un Gauvain érotomane?). Qu'est-ce alors que la « terre dévastée »? Notre présent?

Pour les auteurs qui en réécrivent les récits, le Moyen Âge permettrait in fine une traduction esthétique, en quelque sorte la plus contemporaine, de la condition humaine. Drôle, licencieux, mais sans valeur ni beauté. Terriblement réaliste, somme toute. Chez Dorst, le rire légendaire de Merlin traverse la pièce, celui de Perceval résonne. Pas dans la mise en scène de Dana.

Michèle Gally

Université de Provence - GDR «Théories du poétique »

serait peut-être à réserver à cette représentation, et usage, du médiéval : hétéroclite, vulgaire, sans spiritualité ni pensée. 